\title{
Induced Sputum Analysis in Subjects With Systemic Sclerosis
}

\author{
Irena Litinsky MD, Elizabeth Fireman PhD, Daphna Paran MD, Ari Polachek MD, \\ Adi Broide MD, Amir Sharabi PhD, Marina Anouk MD, and Ori Elkayam MD
}

\begin{abstract}
BACKGROUND: Induced sputum is a noninvasive tool, aimed at collecting cellular and soluble materials from lung airways. Induced sputum sampling analysis has been validated in chronic obstructive lung diseases as well as in various diffuse interstitial lung disorders. Our objective was to evaluate the utility of induced sputum speciments of cellular and soluble materials noninvasively sampled from the lung airways of subjects with systemic sclerosis compared with healthy controls and determine possible correlation with disease manifestations. METHODS: The study population included 20 consecutive subjects with systemic sclerosis and 16 healthy volunteers (controls). All of the participants underwent pulmonary function testing (PFT), single-breath diffusion capacity of carbon monoxide, and induced sputum cytology analysis. RESULTS: The systemic sclerosis subjects' induced sputum samples contained a higher neutrophil count $(P=.09)$ and lower lymphocyte count $(P=.045)$ compared with the controls. Within the systemic sclerosis population, several clinical, PFT, and induced sputum findings were positively correlated: the modified Rodnan skin score score and CD3 $(P=.044)$, modified Rodnan skin score and CD4 $(P=.04)$, modified Rodnan skin score and percentage neutrophils $(P=.059)$, and total lung capacity (TLC) and percentage lymphocytes $(P=.02)$. Induced sputum neutrophil counts were inversely associated with TLC $(P=.02)$ and FVC $(P=.02)$. Induced sputum samples from the subgroup of systemic sclerosis subjects with PFT restrictive pattern contained a significantly lower percentage of lymphocytes $(P=.004)$ and lower $\mathrm{CD} 4 / \mathrm{CD8}$ ratio $(P=.03)$ compared with controls. In this subgroup, TLC also was negatively correlated with the neutrophil count $(P=.043)$, and positive correlations were found between TLC and CD3 $(P=.053)$, disease duration and CD4/CD8 ratio $(P=.033)$, TLC and FVC and percentage macrophages $(P=.033$ and $P=.052$, respectively), diffusion per unit of alveolar volume, and percentage macrophages $(P=.032)$, and $C$-reactive protein and percentage lymphocytes, percentage macrophages, and neutrophil count $(P=.02, P=.008$, and $P=.006$, respectively). CONCLUSIONS: Induced sputum analysis of subjects with systemic sclerosis, including those with restrictive lung disease, shows changes in cellular pattern and correlation with several highly relevant clinical and PFT parameters. Key words: induced sputum; systemic sclerosis; interstitial lung disease; inflammation. [Respir Care 2016;61(10):1369-1373. (C) 2016 Daedalus Enterprises]
\end{abstract}

\section{Introduction}

Systemic sclerosis is a relatively rare multisystem disease characterized by microvascular damage, autoimmu-

Drs Litinsky, Paran, Polachek, Broide, Sharabi, Anouk, and Elkayam are affiliated with the Department of Rheumatology, and Dr Fireman is affiliated with the Institute of Pulmonary and Allergic Diseases, Tel Aviv Sourasky Medical Center, Sackler Faculty of Medicine, Tel Aviv University, Tel Aviv, Israel.

The authors have disclosed no conflicts of interest. nity, and massive collagen overproduction and deposition in the skin and internal organs. ${ }^{1}$ Interstitial lung disease is common among systemic sclerosis patients, with evidence of interstitial changes on high-resolution computed tomography in up to $90 \%$ of systemic sclerosis patients and

Correspondence: Irena Litinsky MD, Department of Rheumatology, Tel Aviv Medical Center, 6 Weizmann Street, Tel Aviv 64239, Israel. E-mail: litinskyira@yahoo.com.

DOI: $10.4187 /$ respcare.04706 
clinically important lung fibrosis in approximately $25 \%$ of all patients with systemic sclerosis. ${ }^{2-4}$

Traditional pulmonary function tests (PFTs) and highresolution computed tomography remain important tools in the diagnosis and monitoring of systemic sclerosis patients with interstitial lung disorder. Bronchoalveolar lavage is an invasive diagnostic tool that detects cellular infiltrate in the alveoli. The procedure is very unpleasant for the patients and requires expensive medical equipment while providing limited prognostic information. ${ }^{5}$ It is therefore not routinely used for pulmonary evaluation in systemic sclerosis. In contrast, induced sputum is a noninvasive tool that can be used to collect cellular and soluble materials from lung airways. It is well accepted by patients, requires inexpensive medical equipment, is safer than bronchoalveolar lavage, and can be repeated as frequently as needed.6,7 Induced sputum sampling has been validated as an approach to study bronchial inflammation in asthma ${ }^{8,9}$ and chronic obstructive lung disorders. ${ }^{10}$ Promising results have been reported in the evaluation of various interstitial lung disorders, including idiopathic type pulmonary fibrosis, ${ }^{11,12}$ sarcoidosis, ${ }^{13}$ hypersensitivity pneumonitis, ${ }^{14}$ and lung involvement in Crohn disease. ${ }^{15}$ Fireman et al $^{16}$ observed a significantly higher percentage of neutrophils and a lower percentage of macrophages in the induced sputum of subjects with idiopathic pulmonary fibrosis.

Evidence of the potential use of induced sputum for the evaluation of inflammation associated with systemic sclerosis is very limited. The purpose of our current study was to explore the possible usefulness of induced sputum cellular analysis in the assessment of systemic sclerosis subjects and to investigate its possible relationship with disease manifestations.

\section{Methods}

The study was approved by the medical ethics committee of Tel Aviv Sourasky Medical Center, and written informed consent was obtained from all participants.

\section{Subjects}

Twenty consecutive patients with systemic sclerosis (19 females and 1 male) who were being treated and followed up at the Department of Rheumatology of the Tel Aviv Sourasky Medical Center (Tel Aviv, Israel) and 16 apparently healthy volunteers participated in this study. Exclusion criteria were the presence of other chronic inflammatory lung disease and acute upper or lower lung disease within the 3 months before study entry.

\section{QUICK LOOK}

\section{Current knowledge}

Induced sputum sampling has been validated as an approach to study bronchial inflammation in asthma and chronic obstructive lung disorders. Promising results have been reported in the evaluation of various interstitial lung disorders, including idiopathic type pulmonary fibrosis. Evidence of the potential use of induced sputum for the evaluation of inflammation associated with systemic sclerosis is very limited.

\section{What this paper contributes to our knowledge}

The induced sputum samples of subjects with systemic sclerosis contained a higher neutrophil count and lower lymphocyte count compared with the healthy controls. Induced sputum analysis of systemic sclerosis subjects, including those with restrictive lung disease, showed changes in cellular pattern and correlation with several highly relevant clinical and pulmonary function testing parameters.

\section{Clinical Assessment}

Clinical assessment was performed on the same day as induced sputum and PFT. Each subject gave a complete past medical history, including the current use of medications, and underwent a physical examination that included the modified Rodnan skin score, presence of digital ulcers, tender and swollen joint count, clinical symptoms (dyspnea, cough, dyspepsia, and dysphagia), and patient and physician disease activity evaluation by a visual activity score (10-100). Laboratory assessment included erythrocyte sedimentation rate and C-reactive protein level.

\section{Pulmonary Function Tests}

PFTs (spirometry, lung volumes, and diffusing capacity) were performed with a Masterlab spirometer (E. Jaeger, Wurzburg, Germany) using standard protocols according to the American Thoracic Society specifications. ${ }^{17}$

\section{Induced Sputum Collection and Processing}

Sputum was induced by inhalation of hypertonic saline generated by a Aerosonic Ultrasonic Nebulizer 5000D/5000I (DeVilbriss-Health Care Corporation, Somerset, Pennsylvania), a method shown to correlate well with bronchoalveolar lavage. ${ }^{18}$ Induced sputum was recovered after a 20-min inhalation of 3\% saline with an ultrasonic nebulizer through a mouthpiece without a valve 
or nose clip. Ten minutes after the start of nebulization and every $5 \mathrm{~min}$ thereafter, the subjects were asked to rinse their nose and mouth with water to minimize contamination of the nasal secretions with saliva. They were encouraged to cough and expectorate sputum into a sterile plastic container. The sputum was always processed within $2 \mathrm{~h}$. The cells in the sputum were dispersed by mechanical disruption in dithiothreitol, cytospins were prepared, and the supernatant was collected for the measurement of fluid phase constituents. CD4/CD8 subsets were identified by fluorescence-activated cell sorting.

\section{Statistical Analysis}

Demographic and clinical parameters of the studied population were summarized, by mean $\pm \mathrm{SD}$ for continuous data and $95 \%$ CI for the mean. Categorical data were summarized by ratio. Despite the small number of subjects, since a normal distribution and $P$ plot were found, differences between groups were evaluated for continuous data by the $t$ test and for categorical data by the chi-square test. Pearson correlation was used to evaluate the association between sputum cytology, PFT tests, and continuous data related to systemic sclerosis. A $p$ value of 0.05 was considered significant.

\section{Results}

\section{Characteristics of the Subjects and Controls}

The demographic data and baseline characteristics of the study population are provided in Table 1 . Thirty-six participants were included in this study: 20 were systemic sclerosis subjects, of whom $13(65 \%)$ had the diffuse type and $7(35 \%)$ had limited type (CREST syndrome). Their results were compared with those of 16 healthy controls. The subject and control groups were similar in sex (mostly women) (see Table 1 ) and age (mean age $52.3 \pm 17.1 \mathrm{y}$ for systemic sclerosis and $57.9 \pm 10.4$ y for controls). The mean disease duration, calculated from the onset of the first non-Raynaud symptom, was $8.7 \pm 9.2$ y. Seven subjects had a restrictive pattern on PFT. None of the participants had pulmonary artery hypertension. At the time of evaluation, 9 subjects $(45 \%)$ were receiving immunosuppressive therapy ( 3 mofetil mycophenolate, 5 methotrexate, and one rituximab).

\section{Cigarette Smoking}

There were 6 smokers in the control group and none in the systemic sclerosis group.

\section{Pulmonary Function}

The total lung capacity (TLC), FVC, and single-breath diffusion capacity of carbon monoxide for alveolar vol-
Table 1. Clinical and Demographic Characteristic of Systemic Sclerosis Subjects and Healthy Controls

\begin{tabular}{|c|c|c|}
\hline Characteristics & $\begin{array}{l}\text { Systemic Sclerosis } \\
\text { Subjects }(n=20)\end{array}$ & $\begin{array}{l}\text { Controls } \\
(n=16)\end{array}$ \\
\hline Age, mean \pm SD y & $52.3 \pm 17.1$ & $57.9 \pm 10.4$ \\
\hline Sex (male/female), $n$ & $1 / 19$ & $3 / 13$ \\
\hline Smokers/non-smokers, $n$ & $0 / 20$ & $6 / 10$ \\
\hline Disease duration, mean \pm SD y & $8.7 \pm 9.2$ & NA \\
\hline Raynaud duration, mean $\pm \mathrm{SD}$ y & $10.3 \pm 9.2$ & NA \\
\hline Diffuse type, $n(\%)$ & $13(65)$ & NA \\
\hline Limited type, $n(\%)$ & $7(35)$ & NA \\
\hline Digital ulcers, $n(\%)$ & $12(60)$ & NA \\
\hline Interstitial lung disease, $n(\%)$ & $8(40)$ & NA \\
\hline $\begin{array}{l}\text { Gastrointestinal tract involvement, } \\
\quad n(\%)\end{array}$ & $14(70)$ & NA \\
\hline Musculoskeletal involvement, $n(\%)$ & $9(45)$ & NA \\
\hline Renal crisis, $n(\%)$ & $1(5)$ & NA \\
\hline Cardiac involvement, $n(\%)$ & $1(5)$ & NA \\
\hline Immunosuppressive treatment, $n(\%)$ & $9(45)$ & NA \\
\hline $\mathrm{mRSS}$, mean $\pm \mathrm{SD}$ & $6.9 \pm 7.18$ & NA \\
\hline $\mathrm{ESR}$, mean $\pm \mathrm{SD} \mathrm{mm} / \mathrm{h}$ & $30.3 \pm 20.9$ & NA \\
\hline $\begin{array}{l}\text { C-reactive protein, mean } \pm \mathrm{SD} \\
\mathrm{mg} / \mathrm{dL}\end{array}$ & $0.6 \pm 0.5$ & NA \\
\hline $\begin{array}{l}\text { PDAI, mean } \pm \text { SD visual activity } \\
\text { score }\end{array}$ & $31.3 \pm 16.6$ & NA \\
\hline $\begin{array}{l}\text { NA = not applicable } \\
\text { mRSS = modified Rodnan skin score } \\
\text { ESR = erythrocyte sedimentation rate } \\
\text { PDAI = patient disease activity evaluation index }\end{array}$ & & \\
\hline
\end{tabular}

Table 2. Comparison of Pulmonary Function Among Systemic Sclerosis Subjects

\begin{tabular}{lccl}
\hline \hline Variable & Diffuse Type $(n=13)$ & Limited Type $(n=7)$ & $P$ \\
\hline TLC & $78.04 \pm 19.57$ & $93.75 \pm 14.15$ & .08 \\
FVC & $78.88 \pm 20.48$ & $93.74 \pm 9.7$ & .041 \\
DLCO/VA & $76.43 \pm 15.87$ & $84.31 \pm 16.93$ & .31 \\
& & \\
Values are mean \pm SD. & & \\
TLC = total lung capacity \\
DLCO/VA = single breath diffusion capacity of carbon monoxide for alveolar volume \\
\end{tabular}

ume results of the systemic sclerosis group were significantly lower than those of the controls $(P<.001, P<.001$, and $P=.003$, respectively).

Subjects with diffuse type systemic sclerosis $(n=13)$ had lower TLC levels and significantly decreased FVC levels compared with the subjects with limited type systemic sclerosis (Table 2).

\section{Induced Sputum}

In all participants, the induction of sputum was well tolerated, and no adverse effects were observed. The in- 
Table 3. Comparison of Induced Sputum Parameters in Systemic Sclerosis Subjects and Healthy Controls

\begin{tabular}{lccl}
\hline \hline \multicolumn{1}{c}{ Variable } & $\begin{array}{c}\text { Systemic Sclerosis Subjects } \\
(n=20)\end{array}$ & $\begin{array}{c}\text { Controls } \\
(n=16)\end{array}$ & $P$ \\
\hline Eosinophils, \% & $1.51 \pm 3.81$ & $1.35 \pm 1.82$ & .87 \\
Neutrophils, \% & $71.36 \pm 19.06$ & $60.26 \pm 17.92$ & .09 \\
Macrophages, \% & $19.75 \pm 15.75$ & $26.05 \pm 16.54$ & .26 \\
Lymphocytes, \% & $6.83 \pm 9.42$ & $12.17 \pm 4.32$ & .045 \\
CD4/CD8 & $3.6 \pm 1.96$ & $2.91 \pm 2.0$ & .33 \\
& & & \\
Values are mean \pm SD. & & & \\
\hline
\end{tabular}

Table 4. Comparison of Induced Sputum Parameters in Systemic Sclerosis Subjects With a Restrictive Pulmonary Function Pattern and Healthy Controls

\begin{tabular}{lccl}
\hline \hline \multirow{1}{c}{ Variable } & $\begin{array}{c}\text { Systemic Sclerosis Subjects } \\
\text { With Restrictive Pulmonary } \\
\text { Function }(n=7)\end{array}$ & Controls & $P$ \\
\hline Eosinophils, \% & $0.42 \pm 0.78$ & $1.35 \pm 1.82$ & .21 \\
Neutrophils, \% & $70.42 \pm 22.86$ & $60.26 \pm 17.92$ & .26 \\
Macrophages, \% & $23.5 \pm 18.79$ & $26.05 \pm 16.54$ & .76 \\
Lymphocytes, \% & $5.57 \pm 4.78$ & $12.17 \pm 4.32$ & .004 \\
CD4/CD8 & $1.67 \pm 0.22$ & $2.91 \pm 2.0$ & .03
\end{tabular}

Values are mean \pm SD

duced sputum of the systemic sclerosis subjects contained a higher neutrophil count $(P=.09)$ and a lower lymphocyte count $(P=.045)$ compared with the control group (Table 3). There was no difference in induced sputum cytology between the various types of systemic sclerosis.

Correlation between induced sputum cellularity and clinical findings revealed a significant positive correlation among several clinical, PFT and induced sputum findings (modified Rodnan skin score and CD3 [ $P=.044]$, modified Rodnan skin score and CD4 [P=.04], and modified Rodnan skin score and percentage neutrophils $[P=.059])$. The neutrophil counts in the subjects' induced sputum were inversely associated with TLC and FVC $(P=.02$ and $P=.002$, respectively).

The induced sputum samples from the subgroup of systemic sclerosis subjects with PFT restrictive pattern contained a significantly lower percentage of lymphocytes $(P=.004)$ and a lower $\mathrm{CD} 4 / \mathrm{CD} 8$ ratio $(P=.03)$ compared with controls (Table 4$)$. The restrictive pattern also correlated positively with the induced sputum cytology count and several clinical, laboratory, and PFT parameters (disease duration and CD4/CD8 ratio $[P=.033]$, TLC and CD3 $[P=.053]$, TLC and FVC and percentage macrophages $[P=.033$ and $P=.052$, respectively $]$, DLCO/VA and percentage macrophages $[P=.032]$, and $\mathrm{C}$-reactive protein and percentage lymphocytes, percentage macro- phages, and neutrophils $[P=.02, P=.008$, and $P=.006$, respectively]). There was a negative correlation between TLC and the neutrophil count $(P=.043)$.

The induced sputum from subjects with interstitial lung disease contained a statistically significantly lower CD4/CD8 ratio then those without interstitial lung disease $(2.81 \pm 2.55$ and $4.13 \pm 1.37$, respectively, $P=.05)$. No significant differences were found in the other induced sputum parameters. The same trend for CD4/CD8 ratio was observed in the subgroup of systemic sclerosis subjects receiving immunosuppressive treatment compared with non-treated subjects $(2.87 \pm 1.15$ and $4.44 \pm 2.4$, respectively). Finally, there was no difference in induced sputum cytology content between the subjects with an obstructive pattern and the controls.

\section{Discussion}

Lung involvement is one of the leading causes of morbidity and mortality in systemic sclerosis. ${ }^{19}$ Early diagnosis of pulmonary manifestations and additional tools for monitoring them in systemic sclerosis are unmet needs. The use of induced sputum, a noninvasive diagnostic procedure, is well established in detecting alveolitis secondary to several inflammatory lung diseases. The role of induced sputum analysis in the assessment of systemic sclerosis patients, however, is not well defined. We evaluated the cellular profile and the clinical utility of the induced sputum procedure in subjects with systemic sclerosis with and without pulmonary involvement and compared the results with those of healthy controls.

The induced sputum analyses revealed lower lymphocyte and higher neutrophil counts in the samples of the subjects with systemic sclerosis compared with the controls. Abnormal findings, including lower lymphocyte counts and reduced CD4/CD8 ratios, were also prominent in the subgroup of systemic sclerosis subjects with a restrictive PFT pattern compared with controls. We also demonstrated correlations between the induced sputum findings and several clinical parameters, including PFT. Taken together, our results showed that the relatively simple, noninvasive induced sputum methodology is capable of detecting subclinical respiratory airway inflammation in subjects with systemic sclerosis who have no symptoms of respiratory involvement.

Our findings support those of previous studies. Vatrella et $\mathrm{al}^{20}$ found that induced sputum samples from subjects with connective tissue disease contained a significantly higher percentage of neutrophils and a lower percentage of macrophages compared with healthy subjects. Damjanov et $\mathrm{al}^{21}$ reported a higher percentage of neutrophils, lymphocytes, and eosinophils in the induced sputum of subjects with systemic sclerosis compared with healthy controls. 
Sánchez-Vidaurre et al ${ }^{22}$ evaluated induced sputum of 25 subjects with systemic sclerosis and found a high percentage of neutrophils in the sputum and significant negative correlation between sputum total cell count and single-breath diffusion capacity of carbon monoxide regardless of the presence of signs of pulmonary involvement.

Yilmaz et $\mathrm{al}^{23}$ also reported an increase in mean macrophage and lymphocyte counts in the induced sputum of 20 subjects with systemic sclerosis compared with the control group, but unlike in other studies, including ours, they demonstrated a decreased number of neutrophils in the induced sputum of subjects with systemic sclerosis in comparison with the control group. In addition, in our study, we also found correlations between clinical and lung functional parameters and induced sputum findings in the total patient population and in those with restrictive PFT pattern.

\section{Conclusions}

Noninvasive induced sputum analysis of subjects with systemic sclerosis reveals changes in cellular patterns and correlations with several selected clinical and PFT parameters. It can be useful as a complementary method for identifying airway inflammation in patients with systemic sclerosis. Future studies are needed with a larger number of subjects to validate the role of induced sputum analysis in the detection and evaluation of patients with systemic sclerosis.

\section{ACKNOWLEDGMENTS}

We thank Esther Eshkol for editorial assistance.

\section{REFERENCES}

1. Tamby MC, Chanseaud Y, Guillevin L, Mouthon L. New insights into the pathogenesis of systemic sclerosis. Autoimmun Rev 2003; 2(3):152-157.

2. Steen VD, Owens GR, Fino GJ, Rodnan GP, Medsger TA Jr. Pulmonary involvement in systemic sclerosis (scleroderma). Arthritis Rheum 1985;28(7):759-767.

3. Steen VD, Conte C, Owens GR, Medsger TA Jr. Severe restrictive lung disease in systemic sclerosis. Arthritis Rheum 1994;37(9):12831289.

4. Cossio M, Menon Y, Wilson W, deBoisblanc BP. Life-threatening complication of systemic sclerosis. Crit Care Clin 2002;18(4):819839.

5. Goh NS, Veeraraghavan S, Desai SR, Cramer D, Hansell DM, Denton $\mathrm{CP}$, et al. Bronchoalveolar lavage cellular profiles in patients with systemic sclerosis-associated interstitial lung disease are not predictive of disease progression. Arthritis Rheum 2007;56(6):20052012.

6. Maestrelli P, Saetta M, Di Stefano A, Calcagni PG, Turato G, Ruggieri MP, et al. Comparison of leukocyte counts in sputum, bronchial biopsies and bronchoalveolar lavage. Am J Respir Crit Care Med 1995;152(6 Pt 1):1926-1931.

7. de la Fuente PT, Romagnoli M, Godard P, Bousquet J, Chanez P. Safety of inducing sputum in patients with asthma of varying severity. Am J Respir Crit Care Med 1998;157(4 Pt 1):1127-1130.

8. Fahy JV, Wong H, Liu J, Boushey HA. Comparison of samples collected by sputum induction and bronchoscopy from asthmatics and healthy subjects. Am J Respir Crit Care Med 1995;152(1):53-58.

9. Beier J, Beeh KM, Semmler D, Beike N, Buhl R. Increased concentrations of glutathione in induced sputum of patients with mild to moderate allergic asthma. Ann Allergy Asthma Immunol 2004;92(4): 459-463.

10. Beeh KM, Beier J, Koppenhoefer N, Buhl R. Increased glutathione disulfide and nitrosothiols in sputum supernatant of patients with stable COPD. Chest 2004;126(4):1116-1122.

11. Olivieri D, D'Ippolito R, Chetta A. Induced sputum: diagnostic value in interstitial lung disease. Curr Opin Pulm Med 2000;6(5):411-414.

12. Antoniou KM, Alexandrakis M, Tzanakis N, Tsiligianni I, Tzortzaki EG, Siafakas NM, Bouros DE. Induced sputum versus bronchoalveolar lavage fluid in the evaluation of patients with idiopathic pulmonary fibrosis. Respiration 2005;72(1):32-38.

13. D'Ippolito R, Foresi A, Chetta A, Casalini A, Castagnaro A, Leone C, Olivieri D. Induced sputum in patients with newly diagnosed sarcoidosis. Comparison with bronchial wash and BAL. Chest 1999; 115(6):1611-1615.

14. D’Ippolito R, Chetta A, Foresi A, Marangio E, Castagnaro A, Merliniaft $\mathrm{S}$, et al. Induced sputum and bronchoalveolar lavage from patients with hypersensitivity pneumonitis. Respir Med 2004;98(10): 977-983.

15. Fireman Z, Osipov A, Kivity S, Kopelman Y, Sternberg A, Lazarov E, Fireman E. The use of induced sputum in the assessment of pulmonary involvement in Crohn's disease. Am J Gastroenterol 2000; 95(3):730-734.

16. Fireman E, Topilsky I, Greif J, Lerman Y, Schwarz Y, Man A, Topilsky M. Induced sputum compared to bronchoalveolar lavage for evaluating patients with sarcoidosis and non-granulomatous interstitial lung disease. Respir Med 1999;93(11):827-834.

17. American Thoracic Society. ATS Statements: snowbird workshop on standardization of spirometry. Am Rev Respir Dis 1979;119(5): 831-838.

18. Fireman E. Induced sputum: opening a new window to the lung. Sarcoidosis Vasc Diffuse Lung Dis 2001;18(3):263-271.

19. Schoenfeld SR Castelino FV. Interstitial lung disease in scleroderma. Rheum Dis Clin N Am 2015;41(2):237-248.

20. Vatrella A, Bocchino M, Perna F, Scarpa R, Galati D, Spina S, et al. Induced sputum as a tool for early detection of airway inflammation in connective disease-related lung involvement. Respir Med 2007; 101(7):1383-1389.

21. Damjanov N, Ostojic P, Kaloudi O, Alari S, Guiducci S, Stanflin N, et al. Induced sputum in systemic sclerosis interstitial lung disease: comparison to healthy controls and bronchoalveolar lavage. Respiration 2009;78(1):56-62.

22. Sánchez-Vidaurre S, Simeón CP, Cruz MJ, Fonollosa V, Vilardell M, Morell F, Muñoz X. Latent pulmonary inflammation in patients with systemic sclerosis. Arch Bronconeumol 2012;48(1):8-13.

23. Yilmaz N, Abul Y, Bicakcigil M, Golabi P, Celikel C, Karakurt S, Yavuz S. Induced sputum as a method for detection of systemic sclerosis-related interstitial lung disease. Rheumatol Int 2012;32(7): 1921-1925. 